\title{
Diagnostic accuracy of non-contrast magnetic resonance angiogram of infra-popliteal arteries prior to fibular-free flap harvest
}

\author{
Charles Elliott $^{1 *}$, Ram Gurajala ${ }^{2}$, Craig Lisicki ${ }^{2}$, Eunice Moon ${ }^{2}$, Karunakaravel Karuppasamy ${ }^{2}$ \\ From 19th Annual SCMR Scientific Sessions \\ Los Angeles, CA, USA. 27-30 January 2016
}

\section{Background}

Patients who are being evaluated to undergo fibula free flap transfer often do not have arterial disease. However a significant anatomical variant cannot be clinically excluded. Contrast enhanced magnetic resonance angiogram (CE-MRA) is commonly performed to identify the suitable side to harvest the flap and to exclude unsuitable infra-popliteal arterial anatomy. The aim of this study is to measure the quality and accuracy of a noncontrast MRA (NC-MRA) technique (Native SPACE = Non-contrast Angiography of the Arteries and Veins using Sampling Perfection with Application Optimized Contrast by using different flip angle Evolution) compared to CE-MRA.

\section{Methods}

Institutional review board approval was obtained for this study. Between October 2012 and August 2014, 16 patients underwent NC-MRA followed by CE-MRA to identify infra-popliteal arterial anatomy prior to fibular-free flap surgery. Clinically, none had symptoms of peripheral vascular disease. CE-MRA was performed in early and later arterial phases. After acquisition of one NC-MRA, it was repeated with different trigger delay at the discretion of the technologist to improve the quality. In this study, NC- and CE-MRA were randomly reviewed and their qualities were recorded on a 3-point scale for each leg: optimal (arteries and any significant disease are clearly detectable), suboptimal (arteries are detectable; however any significant disease cannot be excluded) and non-diagnostic (arteries are not detectable). Using CEMRA as gold standard, sensitivity, specificity, positive

\footnotetext{
${ }^{1}$ School of Medicine, Case Western Reserve University, Cleveland, OH, USA
} Full list of author information is available at the end of the article and negative predictive values of NC-MRA in identifying the type of infra-popliteal arterial anatomy and significant disease (occlusion or $>50 \%$ stenosis) was calculated.

\section{Results}

Compared to 2-phase CE-MRA, an average 1.94 (SD 0.97) number of NC-MRA acquisitions were performed per patient. CE-MRA was optimal in all the legs $(\mathrm{n}=$ 32 ) and NC-MRA was optimal in $75 \%$. NC-MRA was suboptimal in $19 \%$ and non-diagnostic in $6 \%$.

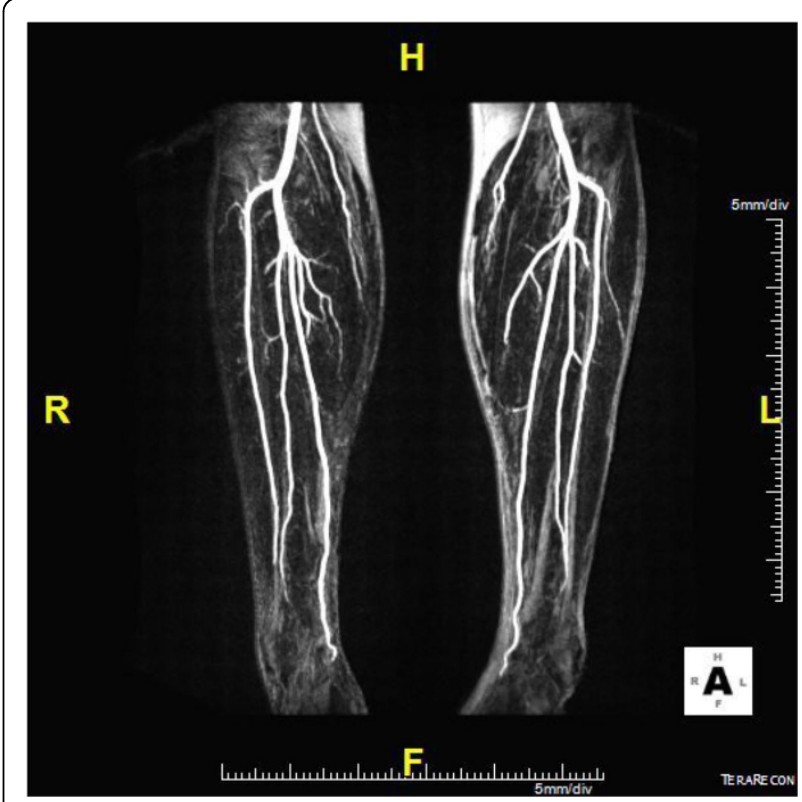

Figure 1 Maximum intensity projection image of the subtracted data of two CE-MRA sequences: One obtained before and one obtained after adminstration of Gadolinium based contrast agent timed to arterial phase. 


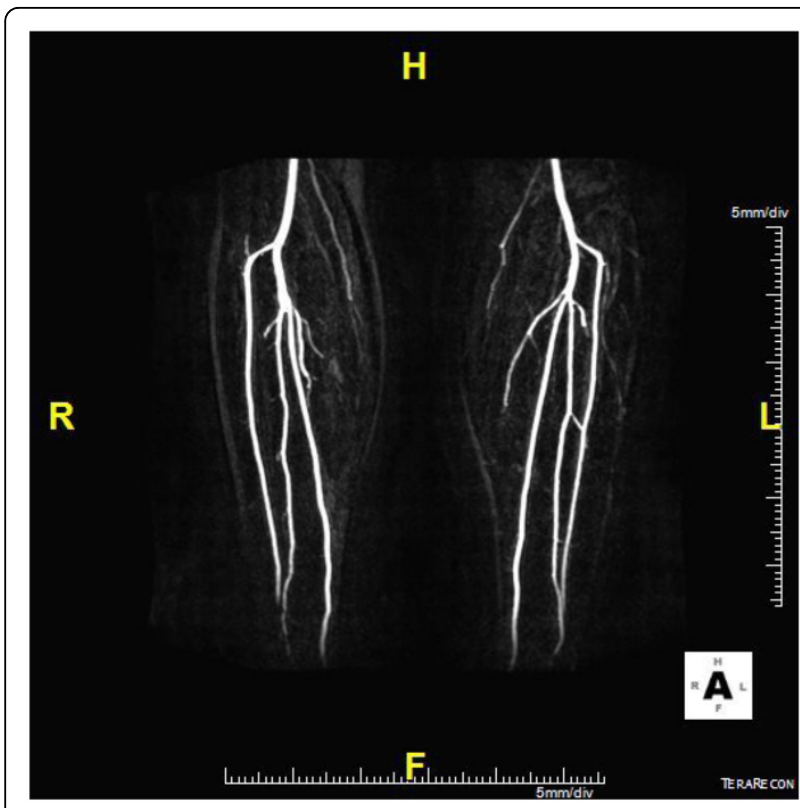

Figure 2 Maximum intensity projection image of the subtracted data of two acqusitions within a Native SPACE sequence obtained when blood flow is minimal and maximal using different ECG trigger times.

NC-MRA correctly identified normal and variant anatomy in $100 \%$ of optimal and suboptimal groups. Among the optimal quality NC-MRA (12 patients/69 infra-popliteal arteries), 58\% demonstrated no significant disease. Compared with CE-MRA, the sensitivity, specificity, positive and negative predictive values of NC-MRA in identifying significant disease was $100 \%$, $89 \%, 30 \%$ and $100 \%$ respectively.

\section{Conclusions}

In patients undergoing MRA prior to fibular-free flap harvest, NC-MRA often provides optimal quality images of infra-popliteal arteries with high negative predictive value for significant disease. Administration of contrast to acquire CE-MRA should be reserved for those with suboptimal or non-diagnostic NC-MRA and when disease is suspected in an optimal quality NC-MRA.

\section{Authors' details}

'School of Medicine, Case Western Reserve University, Cleveland, OH, USA.

${ }^{2}$ Radiology, Cleveland Clinic, Cleveland, OH, USA.

Published: 27 January 2016

doi:10.1186/1532-429X-18-S1-P354

Cite this article as: Elliott et al: Diagnostic accuracy of non-contrast magnetic resonance angiogram of infra-popliteal arteries prior to fibular-free flap harvest. Journal of Cardiovascular Magnetic Resonance 2016 18(Suppl 1):P354.

\section{Submit your next manuscript to BioMed Central} and take full advantage of:

- Convenient online submission

- Thorough peer review

- No space constraints or color figure charges

- Immediate publication on acceptance

- Inclusion in PubMed, CAS, Scopus and Google Scholar

- Research which is freely available for redistribution
Biomed Central 